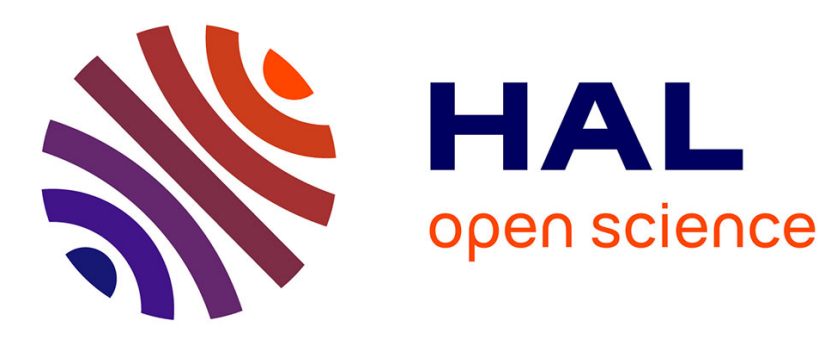

\title{
Binary rototranslational hyper-Rayleigh spectra of H2-He gas mixture
}

Jean-Luc Godet, Tadeusz Bancewicz, Waldemar Glaz, George Maroulis, Anastasios Haskopoulos

\section{- To cite this version:}

Jean-Luc Godet, Tadeusz Bancewicz, Waldemar Glaz, George Maroulis, Anastasios Haskopoulos. Binary rototranslational hyper-Rayleigh spectra of H2-He gas mixture. Journal of Chemical Physics, 2009, 131 (20), pp.204305. 10.1063/1.3264691 . hal-03423703

\section{HAL Id: hal-03423703 \\ https://univ-angers.hal.science/hal-03423703}

Submitted on 10 Nov 2021

HAL is a multi-disciplinary open access archive for the deposit and dissemination of scientific research documents, whether they are published or not. The documents may come from teaching and research institutions in France or abroad, or from public or private research centers.
L'archive ouverte pluridisciplinaire HAL, est destinée au dépôt et à la diffusion de documents scientifiques de niveau recherche, publiés ou non, émanant des établissements d'enseignement et de recherche français ou étrangers, des laboratoires publics ou privés. 


\section{A Ihe Journal of Chemical Physics}

\section{Binary rototranslational hyper-Rayleigh spectra of $\mathbf{H} 2$ - He gas mixture}

J.-L. Godet, T. Bancewicz, W. Głaz, G. Maroulis, and A. Haskopoulos

Citation: The Journal of Chemical Physics 131, 204305 (2009); doi: 10.1063/1.3264691

View online: http://dx.doi.org/10.1063/1.3264691

View Table of Contents: http://scitation.aip.org/content/aip/journal/jcp/131/20?ver=pdfcov

Published by the AIP Publishing

\section{Articles you may be interested in}

Morphology of collisional nonlinear spectra in $\mathrm{H} 2-\mathrm{Kr}$ and $\mathrm{H} 2-\mathrm{Xe}$ mixtures

J. Chem. Phys. 138, 124307 (2013); 10.1063/1.4795438

Collision-induced hyper-Rayleigh spectrum of $\mathrm{H} 2-\mathrm{Ar}$ gas mixture

J. Chem. Phys. 129, 124306 (2008); 10.1063/1.2981042

Moments of hyper-Rayleigh spectra of selected rare gas mixtures

J. Chem. Phys. 127, 134308 (2007); 10.1063/1.2772262

Hyper-Rayleigh spectral intensities of gaseous $\mathrm{Kr}-\mathrm{Xe}$ mixture

J. Chem. Phys. 122, 224323 (2005); 10.1063/1.1925267

The hyper-Rayleigh light scattering spectrum of gaseous $\mathrm{Ne}-\mathrm{Ar}$ mixture

J. Chem. Phys. 118, 6264 (2003); 10.1063/1.1557524

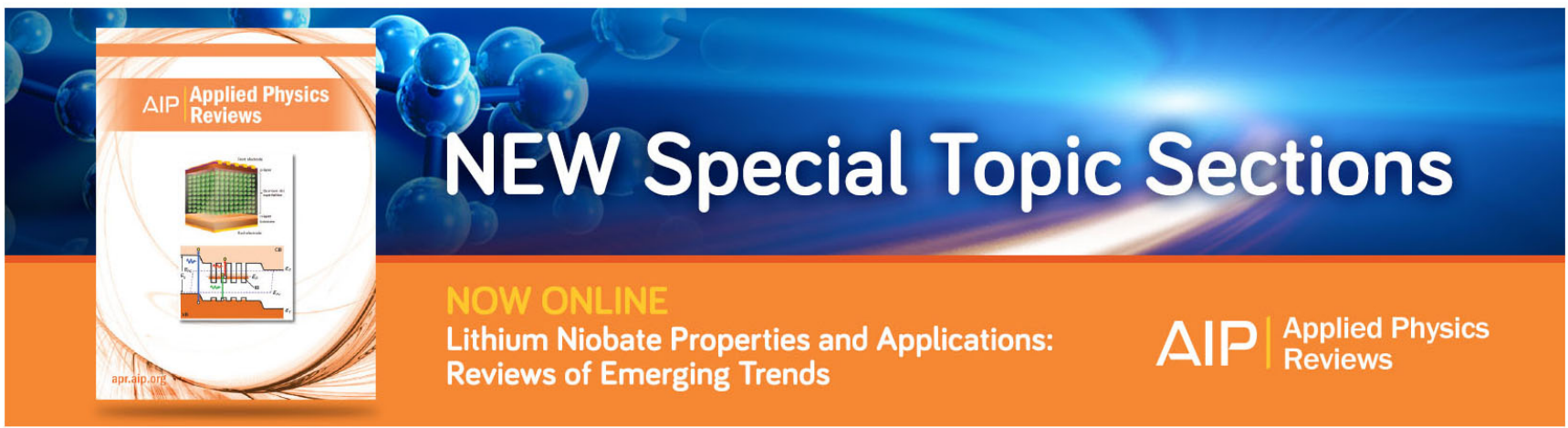




\title{
Binary rototranslational hyper-Rayleigh spectra of $\mathbf{H}_{\mathbf{2}}-\mathrm{He}$ gas mixture
}

\author{
J.-L. Godet, ${ }^{1, a)}$ T. Bancewicz,, ${ }^{2, b)}$ W. Głaz, ${ }^{2, c)}$ G. Maroulis, ${ }^{3, d)}$ and A. Haskopoulos ${ }^{3}$ \\ ${ }^{1}$ Laboratoire des Propriétés Optiques des Matériaux et Applications, Université d'Angers, \\ 2 Boulevard Lavoisier, Angers 49045, France \\ ${ }^{2}$ Nonlinear Optics Division, Faculty of Physics, Adam Mickiewicz University, Poznań 61-614, Poland \\ ${ }^{3}$ Department of Chemistry, University of Patras, Patras GR-26500, Greece
}

(Received 9 September 2009; accepted 27 October 2009; published online 23 November 2009)

\begin{abstract}
The collision-induced rototranslational hyper-Rayleigh spectra of gaseous $\mathrm{H}_{2}-\mathrm{He}$ mixture are computed and discussed in the binary regime. As the input data we use our ab initio computed $\mathrm{H}_{2}-\mathrm{He}$ collision-induced first dipole hyperpolarizability tensor $\Delta \boldsymbol{\beta}(R)$. Both the vector and the septor part of the $\mathrm{H}_{2}-\mathrm{He}$ hyper-Rayleigh spectra are evaluated at room temperature ( $T=295 \mathrm{~K}$ ). The spectra are calculated assuming the full quantum computations based on the Schrödinger equation of the relative translational motion in the isotropic $\mathrm{H}_{2}-\mathrm{He}$ potential as well as using semiclassical methods. (C) 2009 American Institute of Physics. [doi:10.1063/1.3264691]
\end{abstract}

\section{INTRODUCTION}

Dihydrogen-rare gas systems attracted experimental and theoretical attention in recent years. ${ }^{1}$ In particular, the emergence of novel fields as microsolvation in helium clusters ${ }^{2}$ or, predominantly, the spectroscopy of atoms and molecules in liquid helium drops ${ }^{3-5}$ added new impetus to the study of helium-atom or helium-molecule systems. It is worth noting that the collision-induced spectroscopy of the dihydrogenhelium pair is of importance because of its relevance to the molecular astrophysics of planetary atmospheres. ${ }^{6}$ The observation of the collision-induced absorption rototranslational band of this system motivated systematic computational studies of its potential energy surface and the interaction-induced dipole moment. ${ }^{7}$ Recently we reported the first study of the collision-induced hyper-Rayleigh (CIHR) spectrum of the dihydrogen-argon system. ${ }^{8,9}$ The aim of this paper is to extend previous efforts to the determination of the CIHR spectrum of the dihydrogen-helium pair, thus enriching our fundamental knowledge of this important system.

We consider a collision-induced complex composed of a linear centrosymmetric molecule and an atom-like $\mathrm{H}_{2}-\mathrm{He}$. Every monomer of this complex has a center of symmetry. In such monomers, the electric properties described by the odd rank tensors are forbidden. ${ }^{10-13}$ However, as a result of intermolecular interactions (collisions) between monomers, a short-life supermolecule is produced, having configurations which do not possess a center of symmetry. In such a supermolecule, some phenomena which were forbidden in individual monomers are allowed. The incident laser radiation can induce rotational Raman transitions in $\mathrm{H}_{2}$. These ones are combined with the rototranslational transitions appearing due to the effect of the relative rototranslational movement

\footnotetext{
a)Electronic mail: jean-luc.godet@univ-angers.fr.

${ }^{b)}$ Electronic mail: tbancewi@zon12.physd.amu.edu.pl. URL: http:// zon8.physd.amu.edu.pl/ tbancewi.

${ }^{c}$ Electronic mail: glaz@kielich.amu.edu.pl.

${ }^{\mathrm{d})}$ Electronic mail: maroulis@upatras.gr
}

of the atom with respect to the molecule. For instance in $\mathrm{H}_{2}-\mathrm{He}$, the electromagnetic radiation is absorbed by the dipole moment which is collision-induced. ${ }^{14,15}$ Similarly, the third rank tensor of the first dipole hyperpolarizability $\boldsymbol{\beta}$ responsible for the hyper-Rayleigh scattering of light is inconsistent with any centrosymmetic microsystem. The collision induced first dipole hyperpolarizability tensor $\Delta \boldsymbol{\beta}(R)$ appears however as a result of the intermolecular interaction. ${ }^{16}$ In the paper, we discuss the CIHR binary spectra of the $\mathrm{H}_{2}-\mathrm{He}$ mixture. We lean heavily on an recent, exhaustive computational study of the interaction electric dipole moment, polarizability, and hyperpolarizability of the helium-dihydrogen pair. $^{17}$

Hyper-Rayleigh and hyper-Raman spectroscopy has long history since the early work of Kielich, ${ }^{16,18}$ Maker and co-workers, ${ }^{19,20}$ Cyvin et al. ${ }^{21}$ and Long. ${ }^{22}$ Recently, experimental method development opened new possibilities in this field due to microspectroscopic methods and substantial improvements in the detection of very low optical signals. ${ }^{23,24}$

\section{SYMMETRY ADAPTED COMPONENTS OF THE COLLISION-INDUCED PAIR HYPERPOLARIZABILITY OF $\mathrm{H}_{2}-\mathrm{He}$}

The ab initio Cartesian components of the collisioninduced hyperpolarizability of the $\mathrm{H}_{2}-\mathrm{He}$ pair are given in Tables I and II (see Ref. 17 for more details). In our computations, the internuclear distance $R_{\mathrm{H}-\mathrm{H}}$ is equal to $1.449 a_{0}$.

Here the sets of equations ${ }^{9}$ for the collision-induced hyperpolarizability components (for the detailed discussion, see also Ref. 25),

$$
\begin{aligned}
\Delta \beta_{\mu}^{(K)}(R)= & \left(\frac{4 \pi}{2 K+1}\right)^{1 / 2} \sum_{\lambda L}(2 L+1)^{1 / 2} \\
& \times \Delta \beta_{\lambda L}^{(K)}(R) Y_{\lambda \mu}(\Omega) C_{\lambda \mu L 0}^{K \mu},
\end{aligned}
$$

have been inverted to allow computation of the symmetry adapted components $\Delta \beta_{\lambda L}^{(K)}(R)$ for the vector-like $(K=1)$ and the septor-like $(K=3)$ part of the collision-induced $\mathrm{H}_{2}-\mathrm{He}$ hyperpolarizability tensor. There are certain restrictions on 
TABLE I. The Cartesian components of the $\mathrm{H}_{2}-\mathrm{He}$ collision-induced hyperpolarizability for the $\mathrm{L}$ shape configuration (left panel) and the $\mathrm{T}$ shape configuration (right panel). All data are given in atomic units.

\begin{tabular}{rcrccc}
\hline \hline \multicolumn{1}{c}{$R$} & $\Delta \beta_{x x z}(R)$ & $\Delta \beta_{z z z}(R)$ & $\Delta \beta_{x x x}(R)$ & $\Delta \beta_{x y y}(R)$ & $\Delta \beta_{x z z}(R)$ \\
\hline 3.0 & 7.12 & 20.61 & 13.38 & 4.73 & 5.26 \\
3.5 & 4.21 & 16.64 & 10.41 & 2.94 & 3.24 \\
4.0 & 2.61 & 12.30 & 7.65 & 1.87 & 2.06 \\
4.5 & 1.63 & 8.43 & 5.22 & 1.18 & 1.31 \\
5.0 & 1.02 & 5.36 & 3.29 & 0.74 & 0.83 \\
5.5 & 0.63 & 3.14 & 1.90 & 0.46 & 0.53 \\
6.0 & 0.40 & 1.69 & 0.99 & 0.29 & 0.34 \\
7.0 & 0.17 & 0.33 & 0.15 & 0.13 & 0.16 \\
8.0 & 0.09 & -0.04 & -0.06 & 0.07 & 0.09 \\
9.0 & 0.05 & -0.09 & -0.08 & 0.04 & 0.05 \\
10.0 & 0.03 & -0.07 & -0.06 & 0.03 & 0.03 \\
11.0 & 0.02 & -0.05 & -0.04 & 0.02 & 0.02 \\
12.0 & 0.02 & -0.04 & -0.03 & 0.01 & 0.02 \\
\hline \hline
\end{tabular}

the symmetry adapted coefficients $\Delta \beta_{\lambda L}^{(K)}(R)$ of Eq. (1) imposed by the geometry of the system. ${ }^{9}$ For the hyperpolarizability tensor $(K=1$ and/or 3$), \lambda+L$ must be odd. On the other hand for the $\mathrm{H}_{2}$ (homonuclear) molecule $\lambda$ must be even $^{15}(\lambda=0,2,4, \cdots)$. Another restriction results from the Clebsch-Gordan coefficient $C_{\lambda \mu L 0}^{K \mu}$ in Eq. (1). For the vector part $(K=1)$, only the relations $\lambda=L-1$ and/or $\lambda=L+1$ are allowed. For the septor part the following relations are possible: $\lambda=L-3, \lambda=L-1, \lambda=L+1, \lambda=L+3$. From the $a b$ initio Cartesian collision-induced hyperpolarizability components computed for $\mathrm{He}$ located on an axis at $0^{\circ}, 45^{\circ}$, and $90^{\circ}$ in respect to the internuclear axis of $\mathrm{H}_{2}$, we calculate, solving Eq. (1), four ab initio vector-type $(K=1)$ symmetry adapted components with $\lambda L$ indices equal to $01,23,25$, and 45 . We neglect the 43 symmetry-adapted component following the arguments from Ref. 15. These components are given in Fig. 1. For the septor part $(K=3)$ of the $\mathrm{H}_{2}-\mathrm{He}$ collision-induced hyperpolarizability, we compute seven ab initio symmetry adapted components with $\lambda L$ equal to $03,21,23,25,41,43$, and 45. The septor-type components are presented in Fig. 2. These components are suitable in the computation of the CIHR spectrum ${ }^{8,26}$ of the $\mathrm{H}_{2}-\mathrm{He}$ pair since their index $\lambda$

TABLE II. The Cartesian components of the $\mathrm{H}_{2}-\mathrm{He}$ collision-induced hyperpolarizability. The atom of helium is located on a line in $45^{\circ}$ of the $\mathrm{H}-\mathrm{H}$ axis. All data are given in atomic units.

\begin{tabular}{rrrrrrr}
\hline \hline \multicolumn{1}{c}{$R$} & $\Delta \beta_{x x x}(R)$ & $\Delta \beta_{x y y}(R)$ & $\Delta \beta_{x x z}(R)$ & $\Delta \beta_{y y z}(R)$ & $\Delta \beta_{x z z}(R)$ & $\Delta \beta_{z z z}(R)$ \\
\hline 3.0 & 11.51 & 4.00 & 3.79 & 4.24 & 4.08 & 13.53 \\
3.5 & 8.01 & 2.43 & 3.36 & 2.57 & 3.53 & 9.26 \\
4.0 & 5.52 & 1.52 & 2.60 & 1.61 & 2.72 & 6.32 \\
4.5 & 3.66 & 0.96 & 1.82 & 1.01 & 1.89 & 4.17 \\
5.0 & 2.32 & 0.60 & 1.15 & 0.63 & 1.20 & 2.63 \\
5.5 & 1.40 & 0.37 & 0.65 & 0.39 & 0.68 & 1.57 \\
6.0 & 0.81 & 0.24 & 0.33 & 0.25 & 0.34 & 0.90 \\
7.0 & 0.25 & 0.11 & 0.02 & 0.11 & 0.02 & 0.27 \\
8.0 & 0.07 & 0.06 & -0.05 & 0.06 & -0.05 & 0.08 \\
9.0 & 0.03 & 0.03 & -0.05 & 0.03 & -0.05 & 0.02 \\
10.0 & 0.01 & 0.02 & -0.04 & 0.02 & -0.04 & 0.01 \\
11.0 & 0.01 & 0.01 & -0.02 & 0.01 & -0.03 & 0.01 \\
12.0 & 0.01 & 0.01 & -0.02 & 0.01 & -0.02 & 0.00 \\
\hline \hline
\end{tabular}

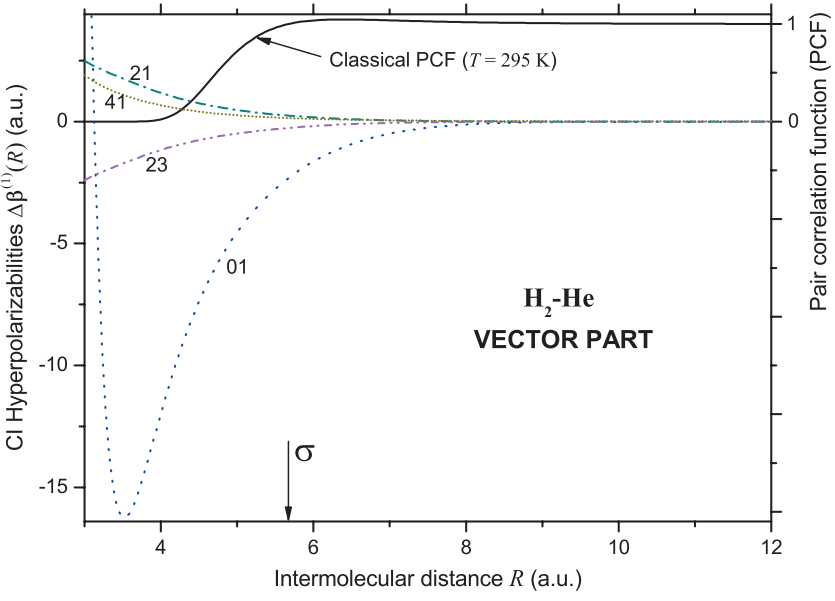

FIG. 1. The $R$ dependence of the $\Delta \beta_{\lambda L}^{(1)}(R)$ irreducible spherical symmetry adapted components of the vector $(K=1)$ part of the collision-induced first dipole hyperpolarizability tensor for $\mathrm{H}_{2}-\mathrm{He}$ vs intermolecular distance $R$, in atomic units. Curves are labeled by $\lambda L$. The collision diameter $\sigma$ of the $\mathrm{H}_{2}-\mathrm{He}$ is marked and the pair correlation function is shown as well.

gives us the selection rules for the rotation motion of $\mathrm{H}_{2}$ molecule whereas the index $L$ leads to selection rules of the relative rotation motion of $\mathrm{He}$ in respect to $\mathrm{H}_{2}$. Accurate $\mathrm{H}_{2}-\mathrm{He}$ intermolecular potential energy surface in also necessary. ${ }^{27,28}$

On the other hand, the asymptotic long-range part of the collision-induced hyperpolarizability can be constructed within the multipolar series. ${ }^{29-31}$ Additional restrictions are imposed then on $\lambda$ and $L$ for multipole-like symmetry adapted components. Certain symmetry-adapted components (mainly of isotropic and anisotropic overlap origin) allowed by Eq. (1) are not possible in the multipolar case. In the case of the long-range multipole first-order collision-induced hyperpolarizability, $L$ is equal to the rank $N$ of the intermolecular interaction tensor $\mathbf{T}_{N}^{(A B)}{ }^{9,31}$ The next multipolar mechanism and the second- and higher-order perturbation theory contributions are also possible but usually give rather small

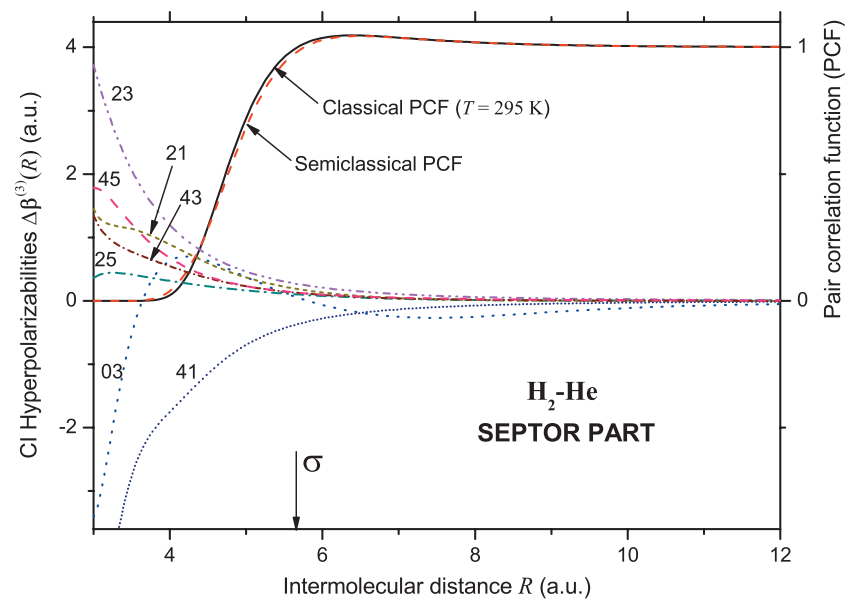

FIG. 2. The $R$ dependence of the $\Delta \beta_{\lambda L}^{(3)}(R)$ irreducible spherical symmetry adapted components of the septor $(K=3)$ part of the collision-induced first dipole hyperpolarizability tensor for $\mathrm{H}_{2}-\mathrm{He}$ vs intermolecular distance $R$, in atomic units. Curves are labeled by $\lambda L$. The collision diameter $\sigma$ of the $\mathrm{H}_{2}-\mathrm{He}$ is marked and the pair correlation function (classical and semiclassical) is shown as well. 
TABLE III. The fitted numerical coefficients of the subsequent spherical components of the dipolar $(K=1)$ part of the collision-induced $\mathrm{H}_{2}-\mathrm{He}$ hyperpolarizabilty. The fitting function is given by Eq. (2). Powers of ten are in parentheses.

\begin{tabular}{|c|c|c|c|c|c|c|c|c|c|}
\hline$\lambda L$ & $a$ & $b$ & $c$ & $d$ & $e$ & $f$ & $g$ & $h$ & $i$ \\
\hline 01 & $-0.702(-3)$ & $0.903(0)$ & $0.368(0)$ & $0.612(-1)$ & $0.681(3)$ & $-0.206(7)$ & $0.600(8)$ & $-0.661(9)$ & $0.261(10)$ \\
\hline 21 & $0.123(-5)$ & $-0.202(-3)$ & $0.159(1)$ & $0.124(0)$ & $-0.116(3)$ & $0.168(6)$ & $-0.424(7)$ & $0.398(8)$ & $-0.131(9)$ \\
\hline 45 & $0.334(-3)$ & $0.120(1)$ & $-0.378(0)$ & $0.192(-4)$ & $-0.261(3)$ & $0.957(5)$ & $-0.205(7)$ & $0.176(8)$ & $-0.551(8)$ \\
\hline
\end{tabular}

(and mainly short-range) corrections. However in the case of the second order induction contribution to the collisioninduced hyperpolarizability ${ }^{30,32}$ two interaction tensors are involved and in general we have contribution of the type $\left(\mathbf{T}_{N_{1}}^{(A B)} \otimes \mathbf{T}_{N_{2}}^{(A B)}\right)_{J}$ where $\left|N_{1}-N_{2}\right| \leq J \leq N_{1}+N_{2}$ and then $L=J$. This shows that for example when $N_{1}=2$ and $N_{2}=3$ the induction contributions with $L=1$ are also possible. The dispersion contributions are also present.

For the collision-induced hyperpolarizability, the lowest value of $L$ equal to three is due to dipole-quadrupole type interaction $(L=1+2)$. In this case, the collision-induced hyperpolarizability components behave like $R^{-4}$. Then for the vector part $(K=1)$ of the collision-induced hyperpolarizability tensor, the components only with $\lambda L$ equal to 23 and 45 have their multipolar counterparts $(\lambda=L-1)$. For the septor part, the multipolar type symmetry adapted components are of the form $\Delta \beta_{03}^{(3)}(R), \Delta \beta_{23}^{(3)}(R), \Delta \beta_{25}^{(3)}(R)$, and $\Delta \beta_{45}^{(3)}(R)(\lambda$ $=L-1$ and $\lambda=L-3)$. We note that the last two components are for $L=5$. The $L=3$ type components result mainly from $\alpha \boldsymbol{B}$ mechanism where the dipole polarizability tensor of $\mathrm{He}$ interacts via the dipole ${ }^{2}$-quadrupole $\boldsymbol{B}$ tensor of $\mathrm{H}_{2}$ together with the mechanism where the $\boldsymbol{B}$ tensor of He interacts with the dipole polarizability of $\mathrm{H}_{2}$. In our discussion we follow the notation of Buckingham. ${ }^{33}$ Within the interaction of the $L=5$ type, $(L=3+2)$, the dipole-octopole polarizability tensor $\boldsymbol{E}$ of $\mathrm{H}_{2}$ molecule interacts with the $\boldsymbol{B}$ tensor of He. But this contribution is expected to be of short range and rather small. The contributions to collision-induced hyperpolarizability from the second hyperpolarizability $\gamma$ of He interacting with the permanent multipole moment $\boldsymbol{Q}$ of $\mathrm{H}_{2}$ are also possible and, in special cases, appear very important (see, e.g., Ref. 34). Kielich et al. ${ }^{35,36}$ attributed almost all CIHR integrated intensity in liquids to the $\gamma \mathbf{T}_{3}^{(A B)} \boldsymbol{Q}$ mechanism. See also Refs. 29-31.
We fit our numerical data with the following function:

$$
\begin{aligned}
\Delta \beta_{\lambda L}^{(K)}(R)= & \left(a R^{2}+b\right) \exp \left(c R-d R^{2}\right) \\
& +\frac{e}{R^{4}}+\frac{f}{R^{7}}+\frac{g}{R^{9}}+\frac{h}{R^{11}}+\frac{i}{R^{13}} .
\end{aligned}
$$

The fitted numerical coefficients of the vector part of the first dipole collision-induced hyperpolarizability of the $\mathrm{H}_{2}-\mathrm{He}$ pair are collected in Table III. Moreover, the fitting numerical coefficients of the septor part are given in Table IV. All data there are given in atomic units.

\section{COLLISION-INDUCED SPECTRUM OF HYPER- RAYLEIGH SCATTERED LIGHT FOR MOLECULAR HYDROGEN-HELIUM SUPERMOLECULAR SYSTEM}

The CIHR spectra have been described extensively in our recent papers. ${ }^{8,37-39}$ Here we present only the most important points. We consider the right angle geometry for observation of the binary $\mathrm{H}_{2}-\mathrm{He}$ CIHR spectra with no analyzer in the experimental setup. We assume the incident laser beam of frequency $\omega$ to be polarized in the scattering plane as well as perpendicularly to this plane. For these conditions, we observe the depolarized $\left(I_{H}\right)$ and the polarized $\left(I_{V}\right)$ CIHR light scattering spectra, respectively. Since the anisotropy of the $\mathrm{H}_{2}-\mathrm{He}$ potential is small, we restrict our considerations to the isotropic potential approximation. ${ }^{26}$ In this case, potential rotational and translational motion are separable. Then, for the double differential cross section for the CIHR depolarized component, we obtain ${ }^{39}$

TABLE IV. The fitted numerical coefficients of the septor $(K=3)$ part of the collision-induced $\mathrm{H}_{2}-\mathrm{He}$ hyperpolarizabilty used in Eq. (2). Powers of ten are

\begin{tabular}{|c|c|c|c|c|c|c|c|c|c|}
\hline$\lambda L$ & $a$ & $b$ & $c$ & $d$ & $e$ & $f$ & $g$ & $h$ & $i$ \\
\hline 03 & $0.215(-5)$ & $-0.143(-2)$ & $0.155(1)$ & $0.115(0)$ & $-0.891(3)$ & $0.550(6)$ & $-0.149(8)$ & $0.145(9)$ & $-0.487(9)$ \\
\hline 21 & $-0.105(-4)$ & $-0.146(-1)$ & $-0.183(-1)$ & $0.117(-1)$ & $0.446(2)$ & $0.319(5)$ & $-0.898(6)$ & $0.870(7)$ & $-0.291(8)$ \\
\hline 23 & $0.126(-4)$ & $-0.733(-3)$ & $0.135(1)$ & $0.119(0)$ & $0.202(3)$ & $0.341(5)$ & $-0.759(6)$ & $0.646(7)$ & $-0.196(8)$ \\
\hline 25 & $0.178(-8)$ & $-0.207(-6)$ & $0.340(1)$ & $0.244(0)$ & $-0.701(2)$ & $0.124(6)$ & $-0.315(7)$ & $0.294(8)$ & $-0.961(8)$ \\
\hline 41 & $-0.161(-2)$ & $-0.506(-1)$ & $0.457(-1)$ & $0.213(-1)$ & $0.292(3)$ & $-0.272(6)$ & $0.716(7)$ & $-0.745(8)$ & $0.283(9)$ \\
\hline 43 & $0.537(-4)$ & $-0.117(0)$ & $-0.347(-1)$ & $0.224(-1)$ & $0.159(3)$ & $0.662(4)$ & $-0.164(6)$ & $0.985(6)$ & $-0.125(7)$ \\
\hline 45 & $0.223(-8)$ & $-0.279(-6)$ & $0.329(1)$ & $0.232(0)$ & $0.134(3)$ & $0.102(5)$ & $-0.153(6)$ & $0.372(6)$ & $0.192(7)$ \\
\hline
\end{tabular}
in parentheses. 


$$
\begin{aligned}
\left(\frac{\partial^{2} I_{H}^{2 \omega_{L}}}{\partial \Omega \partial \omega}\right) / I_{0}^{2}= & \frac{\pi}{2 c} k_{s}^{4}\left\{\frac{2}{45} \sum_{\lambda L} \sum_{j j^{\prime}} P_{j}(2 j+1)\left(2 j^{\prime}+1\right)\right. \\
& \times\left(\begin{array}{ccc}
j & \lambda & j^{\prime} \\
0 & 0 & 0
\end{array}\right)^{2} g_{\lambda L}^{(1)}\left(\omega-\omega_{j j^{\prime}}\right) \\
& +\frac{8}{105} \sum_{\lambda L} \sum_{j j^{\prime}} P_{j}(2 j+1)\left(2 j^{\prime}+1\right) \\
& \left.\times\left(\begin{array}{ccc}
j & \lambda & j^{\prime} \\
0 & 0 & 0
\end{array}\right)^{2} g_{\lambda L}^{(3)}\left(\omega-\omega_{j j^{\prime}}\right)\right\},
\end{aligned}
$$

where $\omega=2 \pi \nu$ stands for the angular frequency shift. The formula corresponding to the polarized intensity $I_{V}$ is analogous to Eq. (3); we only must change the vector part numerical coefficient $2 / 45$ of Eq. (3) to $2 / 9$ and the septor part numerical coefficient $8 / 105$ to $2 / 21$. In Eq. (3), $\left(\begin{array}{lll}j & \lambda & j^{\prime} \\ 0 & 0 & 0\end{array}\right)$ denotes the $3-j$ Wigner symbol, and $P_{j}$ is the Boltzmann population factor. The $g_{\lambda L}^{(K)}(\omega)$ functions appearing in Eq. (3) represent the translational profiles originating from the relative rototranslational motion of the He molecule with respect to the $\mathrm{H}_{2}$ molecule. We note that each translational function is shifted by a frequency of the hydrogen molecule rotational transition $\omega_{j j^{\prime}}$. The translational functions $g_{\lambda L}^{(K)}(\omega)$ have been computed by applying the symmetry adapted components $\Delta \beta_{\lambda L}^{(s, K)}(R)$ of the collision-induced $\mathrm{H}_{2}-\mathrm{He}$ hyperpolarizability tensor presented in Sec. II by the quantum mechanical (QM) as well as semiclassical (SC) methods discussed in details in our previous papers. ${ }^{8,39}$ Here we restrict the presentation to the results of our numerical computations.

\section{NUMERICAL RESULTS AND DISCUSSION}

\section{A. Collision-induced hyperpolarizability}

An inspection of Figs. 1 and 2 shows that the vector part of the collision-induced $\mathrm{H}_{2}-\mathrm{He}$ hyperpolarizability tensor is stronger that its septor part. Among the vector-type components, the isotropic-overlap type component $\Delta \beta_{01}^{(1)}(R)$ plays a predominant role. Moreover, our results show that the exchange and overlap effects are relatively stronger for $\mathrm{H}_{2}-\mathrm{He}$ than for the $\mathrm{H}_{2}-\mathrm{Ar}$ pair studied previously by us. ${ }^{8,9}$ The relative importance of induction and dispersion interactions increases in $\mathrm{H}_{2}-\mathrm{Ar}$ due to the large polarizability and hyperpolarizability of Ar (as the atomic polarizability increases, the relative importance of induction and dispersion interactions increases). ${ }^{30}$ In the same spirit, we observe the diminishing role of the leading multipolelike component $\Delta \beta_{23}^{(1)}$ $\times(R)$ in the vector part of the collision-induced $\mathrm{H}_{2}-\mathrm{He}$ hyperpolarizability, as compared to the $\mathrm{H}_{2}-\mathrm{Ar}$ case. The same holds true for the septor components $\Delta \beta_{03}^{(3)}(R)$ and $\Delta \beta_{23}^{(3)}(R)$.

\section{B. CIHR spectrum}

In this paper we present a comprehensive study of the collision-induced nonlinear first-hyperpolarizability property of the $\mathrm{H}_{2}-\mathrm{He}$ pair and we discuss the CIHR spectrum of this mixture. We computed the CIHR binary spectra of $\mathrm{H}_{2}-\mathrm{He}$ at $T=295 \mathrm{~K}$ by using QM and SC methods described in our previous papers. ${ }^{8,38,39}$ In particular, the SC intensities are ob-

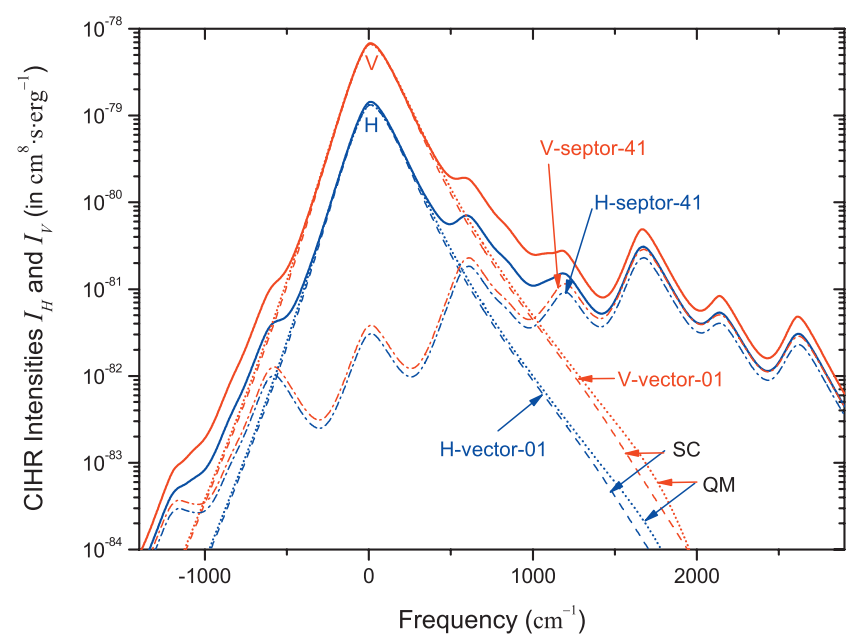

FIG. 3. The CIHR binary polarized $I_{V}\left(V\right.$; in red) and depolarized $I_{H}(H$; in blue) spectra of the $\mathrm{H}_{2}-\mathrm{He}$ pair at $T=295 \mathrm{~K}$ vs frequency shift with regard to the central frequency $4 \pi c / \lambda_{L}$, where $\lambda_{L}=1064 \mathrm{~nm}$ is the exciting laser wavelength. We plot in this figure the resulting $V$ and $H$ spectra (solid lines) as well as two representative $V$ and $H$ subspectra due to the most prominent vector symmetry adapted component $\Delta \beta_{01}^{(1)}(R)$ and due to the septor symmetry adapted hyperpolarizability component $\Delta \beta_{41}^{(3)}(R)$ contributing substantially at far wings. Moreover in the vector case, we show the discrepancies at high frequencies between the QM (dots) and the SC (dashed lines) calculations.

tained by the same way as in Ref. 8 from the classical spectra desymmetrized so as to recover as close as possible the SC spectral moments calculated according to the sum rules within the framework of the SC Wigner-Kirkwood approximation. $^{15}$ It is worthy of note, especially for a pair including a light atom like helium, that the discrepancies between the QM and the SC translational spectra of the vector and septor components are systematically small (a few percent) at low frequencies although they grow in the far wings. However, due to the convolution of the translational contributions with the corresponding rotational stick spectra, these far wings do not contribute significantly to the total spectrum. Therefore, the differences between QM and SC calculations are hardly visible on the final total spectra represented in the logarithmic scale. In Fig. 3, we present our CIHR binary polarized $I_{V}(V)$ and depolarized $I_{H}(H)$ spectra of the $\mathrm{H}_{2}-\mathrm{He}$ pair given in absolute units at $T=295 \mathrm{~K}$ and for an exciting laser wavelength $\lambda_{L}=1064 \mathrm{~nm}$. We plot in this figure the resulting $V$ and $H$ spectra as well as two representative $V$ and $H$ subspectra attributed to the vector symmetry adapted component $\Delta \beta_{01}^{(1)}(R)$ and to the septor symmetry adapted hyperpolarizability component $\Delta \beta_{41}^{(3)}(R)$. We note that the vector part of the collision-induced $\mathrm{H}_{2}-\mathrm{He}$ hyperpolarizability plays the predominant role in shaping the overall (both $V$ and $H$ ) spectra in the low and intermediate frequency range. It is worthy to note that these intensities, mainly related to the 01 vector component, are of the same order of magnitude $\left(>10^{-79} \mathrm{~cm}^{8} \mathrm{~s} \mathrm{erg}^{-1}\right)$ as those of the corresponding $\mathrm{H}_{2}-$ Ar spectra (resulting mainly, in the case of $\mathrm{H}_{2}-\mathrm{Ar}$, from the 03 septor component). ${ }^{8}$ Presumably, this circumstance arises from the strong overlap interaction of $\mathrm{He}$ and $\mathrm{H}_{2}$ and the large 01 component for the $\mathrm{H}_{2}-\mathrm{He}$ pair. Moreover, the contribution brought to the $\mathrm{H}_{2}-\mathrm{He}$ spectra by the septor part could be detected in the far wings. In Fig. 4 we 


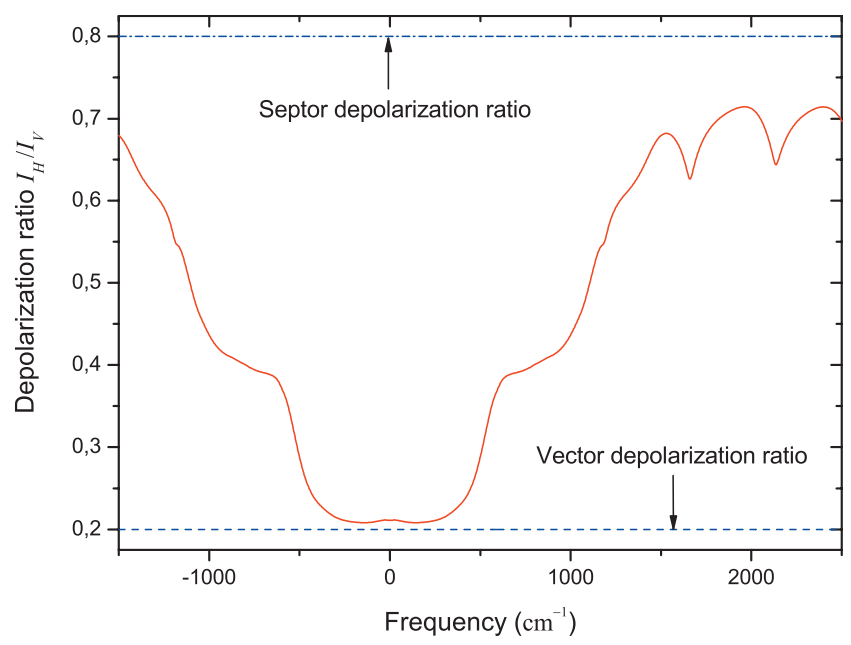

FIG. 4. The depolarization ratio (DR) of the CIHR spectra of $\mathrm{H}_{2}-\mathrm{He}$ vs frequency shift. The DR is very close to its vector part value equal to 0.2 in the low and intermediate frequency range and grows at the high frequency wings, approaching slowly its septor part value equal to 0.8 .

present the depolarization ratio $I_{H} / I_{V}$, which supports very nicely the above conclusions. The depolarization ratio is very close to its vector part value equal to 0.2 in the low and intermediate frequency range and grows at the high frequency wings approaching slowly its septor part value equal to 0.8 .

\section{ACKNOWLEDGMENTS}

We thank Professor Yves Le Duff for the fruitful discussions we had with him about this paper.

\section{APPENDIX: FEASIBILITY OF THE EXPERIMENT}

Our results are given in the absolute units (Fig. 3) and our highest intensity is $I_{V}=6.81 \times 10^{-79} \mathrm{~cm}^{8} \mathrm{~s} \mathrm{erg}^{-1}$ in the vicinity of $0 \mathrm{~cm}^{-1}$. Then, assuming the irradiance of the incident radiation, we can estimate the number of the CIHR photons for a typical scattering experiment. Consider for example a pulsed neodymium-doped yttrium aluminum garnet laser operating at $\lambda_{L}=1064 \mathrm{~nm}$. In the experiments of Pyatt and Shelton ${ }^{40}$ on hyper-Rayleigh scattering in several gas samples, the beam waist radius in the focal plane was $w_{f}$ $=7.5 \mu \mathrm{m}$ and the pulses had a $r=1.5 \mathrm{kHz}$ repetition rate, a $\tau=100 \mathrm{~ns}$ duration and a $\varepsilon=0.7 \mathrm{~mJ}$ energy. We can deduce from these values that the irradiance in the focal plane was ${ }^{41}$

$$
I_{f}=\frac{4 \varepsilon}{\tau}\left(\frac{1}{\pi w_{f}}\right)^{2} \sqrt{\pi \ln (2)}=7,4 \mathrm{GW} \mathrm{cm}^{-2} .
$$

Besides in the case of the CIHR polarized spectrum, the energy scattered near $0 \mathrm{~cm}^{-1}$ per second and per $\mathrm{cm}^{-1}$ is given by

$$
E_{s}=r \tau \Omega_{s} V_{s} n_{\mathrm{H}_{2}} n_{\mathrm{He}} I_{f}^{2} I_{V}(0),
$$

where $V_{s}$ and $\Omega_{s}$ stand for the scattering volume and the solid collection angle, respectively, and $n_{X}$ is the density number of the gas $X$. The scattering volume can be defined as a function of the beam waist and of the confocal parameter $\left(V_{s}=\left(\pi / \lambda_{L}\right)\left(\pi w_{f}^{2}\right)^{2}\right)$. Thus for experimental conditions similar to those described by Pyatt and Shelton, ${ }^{40}$ for a $1: 1$ mixture of $\mathrm{H}_{2}+\mathrm{He}$ both at 30 amagat and considering that only $1 \%$ of the scattered radiation is detected, the CIHR signal is expected to be of $1.1 \times 10^{-3}$ photon per second near $0 \mathrm{~cm}^{-1}$ and per $\mathrm{cm}^{-1}$. This order of magnitude can be compared to the $2 \times 10^{-3} \mathrm{cps}$ reported by Pyatt and Shelton ${ }^{40}$ for hyper-Rayleigh (allowed) intensities. Moreover, Eqs. (A1) and (A2) show that $E_{s} / I_{V}$ (or $E_{s} / I_{H}$ ) is only proportional to $\Omega_{s} n_{\mathrm{H}_{2}} n_{\mathrm{He}} r \varepsilon^{2} / \tau$. The use of higher pump intensities can increase the signal and the signal/noise ratio accordingly, whereas the use of shorter pulse duration times can avoid thermal effects which result from higher intensities. It is also possible to increase the gas pressure, as long as three-body effects can be neglected. This calculation, admittedly rough and approximate, nevertheless suggests that the CIHR experiment, though difficult to perform, is possible.

${ }^{1}$ R. J. Le Roy and J. M. Hutson, J. Chem. Phys. 86, 837 (1987) (and references therein).

${ }^{2}$ E. Bodo, F. Sebastianelli, F. A. Gianturco, and I. Pino, J. Phys. Chem. A 109, 4252 (2005) (and references therein).

${ }^{3}$ J. P. Toennies and A. F. Vilesov, Annu. Rev. Phys. Chem. 49, 1 (1998).

${ }^{4}$ M. Y. Choi, G. E. Douberly, T. M. Falconer, W. K. Lewis, C. M. Lindsay, J. M. Merritt, P. L. Stiles, and R. E. Miller, Int. Rev. Phys. Chem. 25, 15 (2006).

${ }^{5}$ G. D. Scholes and G. R. Fleming, J. Phys. Chem. B 104, 1854 (2000) (and references therein).

${ }^{6}$ L. M. Trafton, in Molecular Complexes in Earth's, Planetary, Cometary and Interstellar Atmospheres, edited by A. A.Vigasin and Z. Slanina (World Scientific, Singapore, 1998).

${ }^{7}$ M. Gustafsson, L. Frommhold, and W. Meyer, J. Chem. Phys. 113, 3641 (2000).

${ }^{8}$ T. Bancewicz, W. Głaz, J.-L. Godet, and G. Maroulis, J. Chem. Phys. 129, 124306 (2008).

${ }^{9}$ T. Bancewicz and G. Maroulis, Phys. Rev. A 79, 044704 (2009).

${ }^{10}$ G. C. Tabisz, in Molecular Spectroscopy (A Specialist Periodical Report), edited by R. F. Barrow, D. A. Long, and J. Sheridan (Chemical Society, London, 1979), Vol. 6, pp. 136-173.

${ }^{11}$ Phenomena Induced by Intermolecular Interactions, NATO ASI Series, edited by G. Birnbaum (Plenum, New York, 1985).

${ }^{12}$ Collision-and Interaction-Induced Spectroscopy, NATO ASI Series C: Mathematical and Physical Sciences, edited by G. C. Tabisz and M. N. Neuman (Kluwer Academic, Dordrecht, 1995), Vol. 452.

${ }^{13}$ J.-M. Hartmann, C. Boulet, and D. Robert, Collisional Effects on Molecular Spectra (Elsevier, Amsterdam, 2008).

${ }^{14}$ G. Birnbaum, S.-I. Chu, A. Delgarno, L. Frommhold, and E. L. Wright, Phys. Rev. A 29, 595 (1984).

${ }^{15}$ L. Frommhold, Collision-Induced Absorption in Gases (Cambridge University Press, Cambridge, 1993).

${ }^{16}$ S. Kielich, Bull. Acad. Polon. Sci., Ser. Sci., Math., Astron. Phys. 12, 53 (1964).

${ }^{17}$ A. Haskopoulos and G. Maroulis, "The interaction electric properties used in the present paper are obtained from CCSD (singles and doubles coupled-cluster) calculations with a flexible, optimized $\mathrm{H} / \mathrm{He}$ $=[6 \mathrm{~s} 4 \mathrm{p} 3 \mathrm{~d} 1 \mathrm{f} / 6 \mathrm{~s} 4 \mathrm{p} 3 \mathrm{~d} 1 \mathrm{f}]$ basis set, Chem. Phys., in press.

${ }^{18}$ S. Kielich, Acta Phys. Pol. 24, 135 (1964).

${ }^{19}$ R. W. Terhune, P. D. Maker, and C. M. Savage, Phys. Rev. Lett. 14, 681 (1965).

${ }^{20}$ P. D. Maker, Phys. Rev. A 1, 923 (1970).

${ }^{21}$ S. J. Cyvin, J. E. Rauch, and J. C. Decius, J. Chem. Phys. 43, 4083 (1965).

${ }^{22}$ D. A. Long, Raman Spectroscopy (McGraw-Hill, New York, 1977).

${ }^{23}$ F. Rachet, M. Chrysos, C. Guillot-Noel, and Y. LeDuff, Phys. Rev. Lett. 84, 2120 (2000).

${ }^{24}$ V. Rodriguez, F. Adamietz, D. Talaga, and J. L. Brunel, Chem. Phys. Lett. 431, 190 (2006).

${ }^{25}$ T. Bancewicz, AIP Conf. Proc. 1108, 108 (2008).

${ }^{26}$ M. Gustafsson, L. Frommhold, X. Li, and K. L. C. Hunt, J. Chem. Phys. 130, 164314 (2009).

${ }^{27}$ A. I. Boothroyd, P. G. Martin, and M. R. Peterson, J. Chem. Phys. 119, 
3187 (2003).

${ }^{28}$ J. Schaefer and W. E. Kohler, Physica 129A, 469 (1985).

${ }^{29}$ A. D. Buckingham, E. P. Concannon, and I. D. Hands, J. Phys. Chem. 98, 10455 (1994).

${ }^{30}$ X. Li, K. L. C. Hunt, J. Pipin, and D. M. Bishop, J. Chem. Phys. 105 , 10954 (1996).

${ }^{31}$ T. Bancewicz, J. Chem. Phys. 111, 7440 (1999).

${ }^{32}$ T. Bancewicz, Mol. Phys. 50, 173 (1983).

${ }^{33}$ A. D. Buckingham, Adv. Chem. Phys. 12, 107 (1967).

${ }^{34}$ T. Bancewicz, AIP Conf. Proc. 963, 250 (2007).

${ }^{35}$ S. Kielich, J. R. Lalanne, and F. B. Martin, Phys. Rev. Lett. 26, 1295
(1971).

${ }^{36}$ S. Kielich, J. R. Lalanne, and F. B. Martin, J. Raman Spectrosc. 1, 119 (1973).

${ }^{37}$ W. Głaz and T. Bancewicz, J. Chem. Phys. 118, 6264 (2003).

${ }^{38}$ W. Głaz, T. Bancewicz, and J.-L. Godet, J. Chem. Phys. 122, 224323 (2005).

${ }^{39}$ W. Głaz, T. Bancewicz, J.-L. Godet, G. Maroulis, and A. Haskopoulos, Phys. Rev. A 73, 042708 (2006)

${ }^{40}$ R. D. Pyatt and D. P. Shelton, J. Chem. Phys. 114, 9938 (2001).

${ }^{41}$ G. Boudebs and K. Fedus, J. Appl. Phys. 105, 103106 (2009). 